\title{
Evaluation of Radio Resource Management Impact on RoF Signal Transmission for Downlink LTE
}

\author{
Pedro Luis Carro ${ }^{(\mathbb{0})}$, Ángela Hernández-Solana ${ }^{(1)}$, and Antonio Valdovinos Bardají
}

\begin{abstract}
Analog radio-over-fiber (RoF) is a suitable technology for efficiently developing the cloud-RAN concept in fifth generation deployments based on long-term evolution advanced (LTE-A). Distortion and radiofrequency (RF) power amplifier gain should be taken into account in order to achieve the desired transmission power at the base station. Both mainly depend on several parameters that characterize the optical link, such as the RF input power and bias current. This paper analyzes the link performance due to variations on the traffic load as expected under real operation. In this paper, we show that it is possible in this scenario to adaptively choose the optimum RF input power to minimize the measured error vector magnitude and reduce the $R F$ subsystem gain. Results show that this adaptation allows to relax the RF amplifier gain requirements up to 3-4 $\mathrm{dB}$ for medium load conditions.
\end{abstract}

Index Terms-Cloud RAN, error vector magnitude, long term evolution, radio over fiber, radio resource management.

\section{INTRODUCTION}

$\mathbf{F}$ UTURE 5G communications systems are based on new proposals which combine a more efficient use of the spectrum and the latest technological advances in signal processing, communications and networks. As far as network deployment is concerned, it provides a higher access element density together with heterogeneity of both access technologies and cell types (macro, micro, pico and femto cells) as a way of increasing capacity. In addition, the Cloud Radio Access Network (C-RAN) concept will be applied [1] on 5G systems, where base station (BS) functionalities are split in two levels: a centralized baseband unit (BBU), of great capacity of computation which includes the Medium Access Control (MAC) and Physical (PHY) layers, and a set of distributed Remote Radio Head (RRH) units where RF transmission and reception are performed, which carry out the opto-electric (transmission) and electro-optic (reception) conversions. One of the biggest challenges in the C-RAN implementation is the design of the link between the BBUs and the RRUs, called fronthaul, which

Manuscript received September 27, 2017; revised November 27, 2017; accepted December 11, 2017. Date of publication December 18, 2017; date of current version February 27, 2018. This work was financially supported by the Spanish Government under Project TEC2014-58341-C4-2-R, MINECO, and FEDER. (Corresponding author: Pedro Luis Carro.)

The authors are with the Department of Electronic Engineering and Communications, Aragon Institute of Engineering Research (I3A), University of Zaragoza, Zaragoza 50018, Spain (e-mail: plcarro@unizar.es; anhersol@unizar. es; toni@unizar.es).

Color versions of one or more of the figures in this paper are available online at http://ieeexplore.ieee.org.

Digital Object Identifier 10.1109/JLT.2017.2784622 should have high bandwidth and low latency. According to those requirements, Radio-over-Fiber (RoF) is a suitable technology for C-RAN fronthaul transmissions as both, high bandwidth and low latency are among its relevant features.

Current Orthogonal Frequency Division Multiplexing (OFDM)-based mobile communication systems like LTE-A are very sensitive to the nonlinear distortions introduced by analogue devices, especially at the transmitter. The power amplifier nonlinear behavior will now add a new source of degradation because of the fiber dispersion and the electric to optical and optical to electric converters [2].

Several studies have analyzed the relationship between the distortion originated in the optical transmission segment between the BBU and the RRH and the RF input power [3]-[6]. Any reduction of power must be compensated for by increasing the RF subsystem gain at the RRH so that the laser does not work near its saturation region. Consequently the RF input power should be as high as possible in order to simplify the amplifier gain requirements. However, when the RF power is greatly reduced, the distortion also increases due to the noise. Thus, if the RF input power is low, the performance of the ana$\log$ RoF link is limited by noise whereas when that power is high, the performance is limited by non-linear distortion. Previous works conclude that a key rule is to choose an optimum $\mathrm{RF}$ input power to minimize these effects and ensure that the laser bias is neither near to the lasing threshold level nor to the saturation region.

Many works focus on the study of the maximum input power which satisfies the EVM requirements for LTE signals established by the 3rd Generation Partnership Project (3GPP) under different parameters, such as the measured constellations Quadrature Phase Shift Keying (QPSK), Quadrature Amplitude Modulation (QAM) (16-QAM, 64-QAM), the input power vs fiber lengths, the carrier aggregation scheme, etc. In [5] an automatic input level controller was needed to achieve the best $E V M$ performance of a downstream RF signal when, besides the optical fiber subsystem (FO), the high power amplifier (HPA) at the input of the antenna is explicitly considered. EVM degradation will be observed at the HPA output if the received power at the RRH drives the amplifier stage out of its linear operation range. Furthermore, these studies consider simplified hypothesis about the downlink LTE-A system operation. In fact, OFDM signals occupying the whole bandwidth of the selected carrier are assumed. However, LTE-A downlink resource patterns and power allocation requirements will have a great impact on a system configuration, as this work explicitly discusses. 


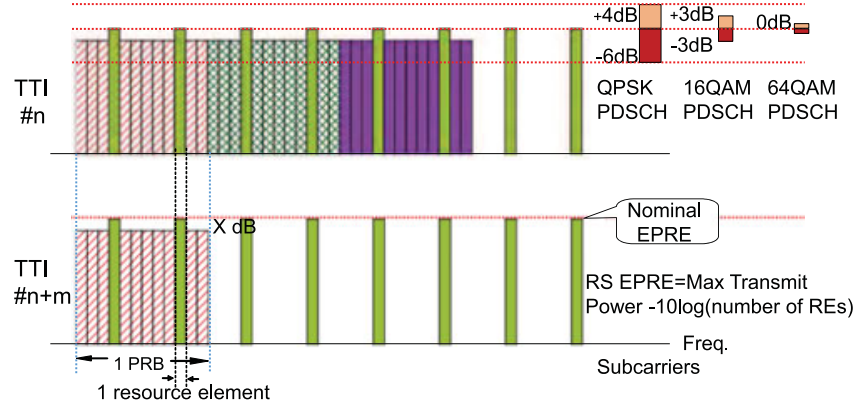

(a)

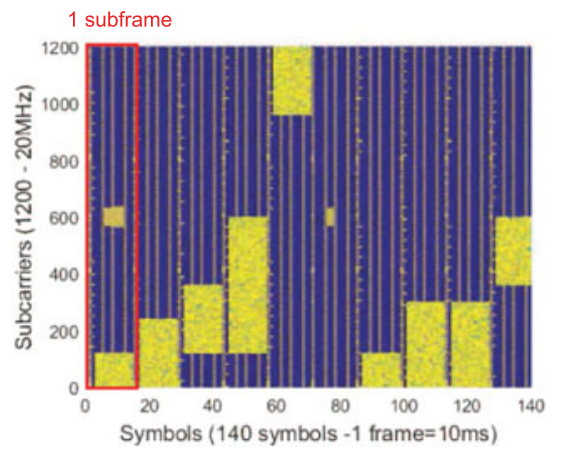

(c)

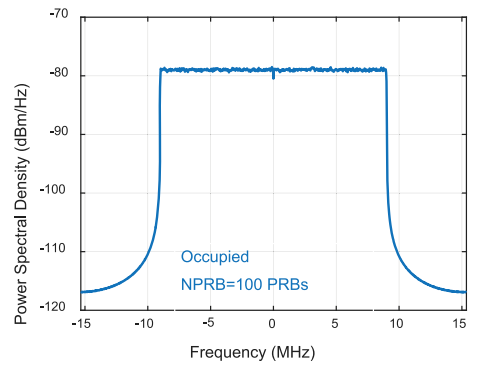

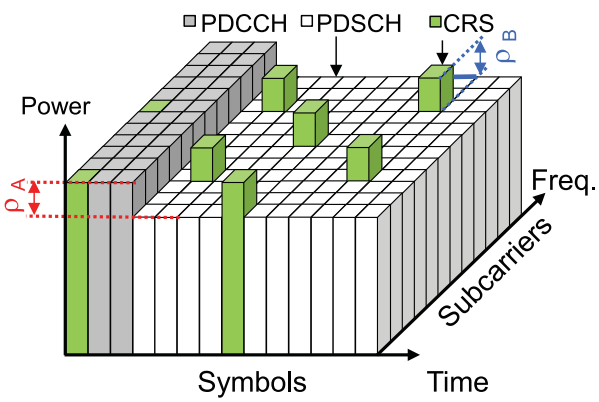

$R S E P R E=$ Reference Signal Energy per RE $P D S C H E P R E=\rho_{B} / \rho_{A} R S E P R E$

(b)

\begin{tabular}{|c|c|c|}
\hline $\begin{array}{c}\text { E-UTRA } \\
\text { channel } \\
\text { bandwidth (MHz) }\end{array}$ & $\begin{array}{c}\text { Number } \\
\text { of PRBs }\end{array}$ & $\begin{array}{c}\text { Total power dynamic } \\
\text { range (dB) }\end{array}$ \\
\hline 1.4 & 6 & 7.7 \\
\hline 3 & 15 & 11.7 \\
\hline 5 & 25 & 13.9 \\
\hline 10 & 50 & 16.9 \\
\hline 15 & 75 & 18.7 \\
\hline 20 & 100 & 20 \\
\hline
\end{tabular}

(d)

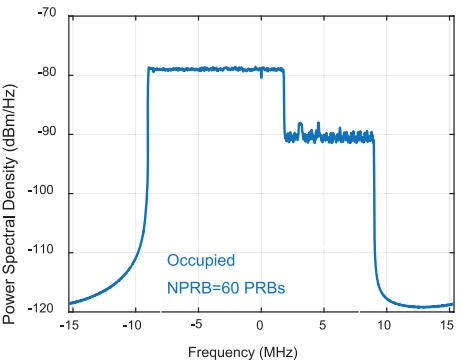

(e)

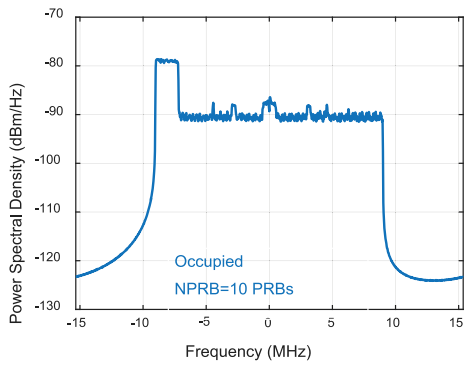

Fig. 1. Resource allocation and downlink power allocation in LTE/LTE-A. (a) Example of evolution of power vs spectral occupation. Definition of E-UTRA BS RE power control dynamic range [7]. (b) Example of downlink power allocation in LTE within a subframe in a PRB. (c) Example of resource allocation in a frame(variable number of occupied PRBs/subframe. (d) E-UTRA BS total power dynamic range [7]. (e) Power Spectral for several PDSCH allocation (NPRBs = 100, 60 and 10). Component Carrier of $20 \mathrm{MHz}$.

This paper is outlined as follows. In Section II, we briefly summarize the basis of radio resource and downlink power allocations in LTE-A and their impact on power variation on the optical link. The analytic signal description is explained in Section III. The experimental setup and the main results are discussed in Section IV. Finally, some conclusions about this work are provided in Section V.

\section{Resource Allocation AND Downlink Power ALLOCATION IN LTE-A}

Downlink power allocation aims at having constant power for all occupied frequency subcarriers or resource elements (RE) in order to better control interference variations at the UEs in intercell scenarios. The RE in eNB is defined as the smallest entity in the resource grid of subcarriers (in the frequency domain) and OFDM symbols (in the time domain). Depending on the power allocation design goals, cell-specific reference signals (CRS), which are embedded into the overall system bandwidth at certain frequency subcarriers $(=\mathrm{RE})$, are transmitted with constant power across the downlink system bandwidth and constant across all subframes (TTI:transmission Time Interval $=1$ subframe $=1 \mathrm{~ms}$ ), as illustrated in Fig. 1(a).

At the air interface, the evolved-NodeB (eNB) sets the default CRS Energy Per RE (RS-EPRE) and calculates it by dividing the maximum allowed output power to the number of subcarriers/REs on the whole bandwidth. For instance, in a bandwidth configuration of $20 \mathrm{MHz}$, the number of REs is 1200 . In this case, if the allowed maximum output power is set to $15 \mathrm{dBm}$, the reference power allocation in an occupied RE which is transmitting a CRS signal is $15 \mathrm{dBm}-10 \log (1200)$. Then, the EPRE of all other signal components or physical channels is set relative to this $R S-E P R E$ value, which is used as a reference. 
The specification includes some feasible power ratio thresholds between the Physical Downlink Control Channel (PDCCH) or Physical Downlink Shared Channel (PDSCH) energy per RE and the RS EPREs. The downlink modulation schemes (QPSK, 16QAM and 64QAM) require different level of EVM accuracy to exploit the full benefit of the modulation. Due to the fact that $E V M$ depends on the power control range, the difference between the maximum and the minimum RE transmitted output power in the case of a specified reference condition is constrained to $X=+4 /-6 \mathrm{~dB},+3 /-3 \mathrm{~dB}$ and $0 \mathrm{~dB}$, for QPSK, 16QAM and 64QAM, respectively, as illustrated in Fig. 1(a) and specified in [7].

On the other hand, the ratio between PDSCH EPRE and the RS EPRE's for each OFDM symbol depend on PDSCH RE's location, and is denoted by either $\rho_{A}$ or $\rho_{B}$. The magnitude $\rho_{B}$ is used if the PDSCH RE lies on the same symbol where there is a RS (index 0,4 ) and the parameter $\rho_{A}$ is used otherwise, according to [7]. $\rho_{A}$ usually corresponds to the parameter $P_{A}$, called reference Signal Power, which is signaled via higher layers. $P_{A}$ allows to set 8 power configurations (ranging from 0 to 7 ), which correspond to $\rho_{A}=\{-6,-4.77,-3,-1.77,0,1,2,3\}$. $\rho_{B}$ is derived as a ratio of $\rho_{A}\left(\rho_{B} / \rho_{A}\right)$. Fig. 1(b) illustrates the EPRE relationships between several signal/physical channels (CRS, PDCCH and PDSCH) in a Physical Resource Block (PRB). A PRB is the minimum unit assigned to an UE in a $1 \mathrm{~ms}$ subframe, consisting of 12 subcarriers $(180 \mathrm{kHz})$ along the whole subframe (14 OFDM symbols), excluding PDCCH. In this work, $P_{A}=4$ implies $\rho_{A}=1$ or $0 \mathrm{~dB}$ and $\rho_{B} / \rho_{A}$ is set to 1. Consequently RS, PDCCH and PDSCH REs are transmitted with the same EPRE.

Besides these few degrees of flexibility, one of the main features of downlink power allocation is the fact that the power per occupied RE remains constant despite any change in the number of resources allocated on the PDSCH. This yields to a dependence of the actual eNB output power on the resource allocation. As a result, the base station transmitted power in a LTE/LTE-A system may severely fluctuate, depending on the number of occupied OFDM subcarriers at any particular time.

It is important to know the requirements which are imposed by the radio resource management strategies defined over the physical layer. In contrast to wired networks, resource allocation in OFDM systems such as LTE-A is fundamentally different. The scheduler at eNB attempts for a suitable resource allocation (power, subcarriers and modulation and coding scheme) between their UEs in each sub-frame. Its decisions are based on several factors such as traffic demands, the quality of service required by the active users, the propagation channel and the interference experienced by each user, the total available power in the enB, the inter-cell interference coordination schemes, etc... Actually, not only the number of PRBs but also the distribution of these PRBs over the carrier bandwidth may change in order to exploit the multiuser multichannel diversity [8]. According to that resource allocation flexibility, the standard [7] defines the power variation dynamic range for different bandwidths [see Fig. 1(d)]. The total power dynamic range is the difference between the maximum and the minimum transmit power in an OFDM symbol that carries
PDSCH and does not contain RS. The dynamic range upper limit is the OFDM symbol power for an eNB at maximum output power (all PRBS are transmitted). The lower bound is the OFDM symbol power when only one PRB is transmitted. For example, when a $20 \mathrm{MHz}$ signal bandwidth is considered, the maximum number of PRBs is 100 . Thus, the dynamic range is $10 \log (100 \mathrm{PRB} / 1 \mathrm{PRB})=20 \mathrm{~dB}$. When $5 \mathrm{MHz}$ is considered, the maximum number of PRBs is 25 , and consequently the dynamic range is $10 \log (25 \mathrm{PRB} / 1 \mathrm{PRB})=13.9 \mathrm{~dB}$. In the remaining symbols, even though one single PRB is transmitted, the dynamic range is limited to $12.58 \mathrm{~dB}$ because some subcarriers are used to transmit cell reference signals, as illustrated in Fig. 1(a) and (c). Finally, power spectral density varies through the time as Fig. 1(e) shows, but EPRE remains constant.

As explained, system operation clearly differs from a usual optical link analysis. At the base station, the RF input power is often selected considering that all PRBs are employed, i.e. under maximum system traffic load conditions. As a result of the actual cell operating situation, which may be far from the previous scenario, the instantaneous load conditions of the cell and the decisions made by the scheduler play a key role on the optical link distortion. From a quantitative point of view, different $E V M$ measurements can be obtained with identical input power values corresponding to different number of allocated PRBs. It is important to pay attention to the fact that the optical link and the RF subsystem must guarantee that downlink power allocation constraints are fulfilled. In terms of ratios between PDCCH, PDSCH and RS-EPRE, they should not vary at the radio interface. Thus, the aim is that the FO+RF subsystem achieved gain remains constant for any distribution of the PRBs occupied in the different sub-frames. Although the output power may actually change every TTI, fluctuations depend on the resource allocation.

\section{SignAl AND SySTEM DESCRIPTION}

The simplest C-RAN architecture with optical fronthaul (Fig. 2) consists of a BBU comprised by the LTE-A signal generator, a semiconductor laser, a dispersive fiber link, and a RRH with a photodetector and a RF amplifier subsystem. For simplicity, we describe the fluctuations on an optically intensity modulation system. With few modifications (those referred to the external modulation systems such as EAMs or MaxZenders), signal variations can be addressed for every particular device, but main conclusions will be still valid.

As far as the OFDM waveform is concerned, optical systems have been classically evaluated using a RF modulated signal with fully filled subcarriers [9], [10]. In this situation, the baseband signal is [11]

$$
x(t)=\sum_{\substack{k=-N / 2 \\ k \neq 0}}^{N / 2} X_{k} e^{j 2 \pi k \Delta f t}=\sum_{\substack{k=-N / 2 \\ k \neq 0}}^{N / 2} X_{k} e^{j \Omega_{k} t}
$$

with $\Omega_{k}=2 \pi k \Delta f$ the subcarrier frequency, $N$ the total number of subcarriers, $\Delta f$ the subcarrier spacing ( $\Delta f=15 \mathrm{KHz}$ in this case) and $X_{k}$ the modulated simbols (containing reference signals and control, synchronization and user data). The laser 


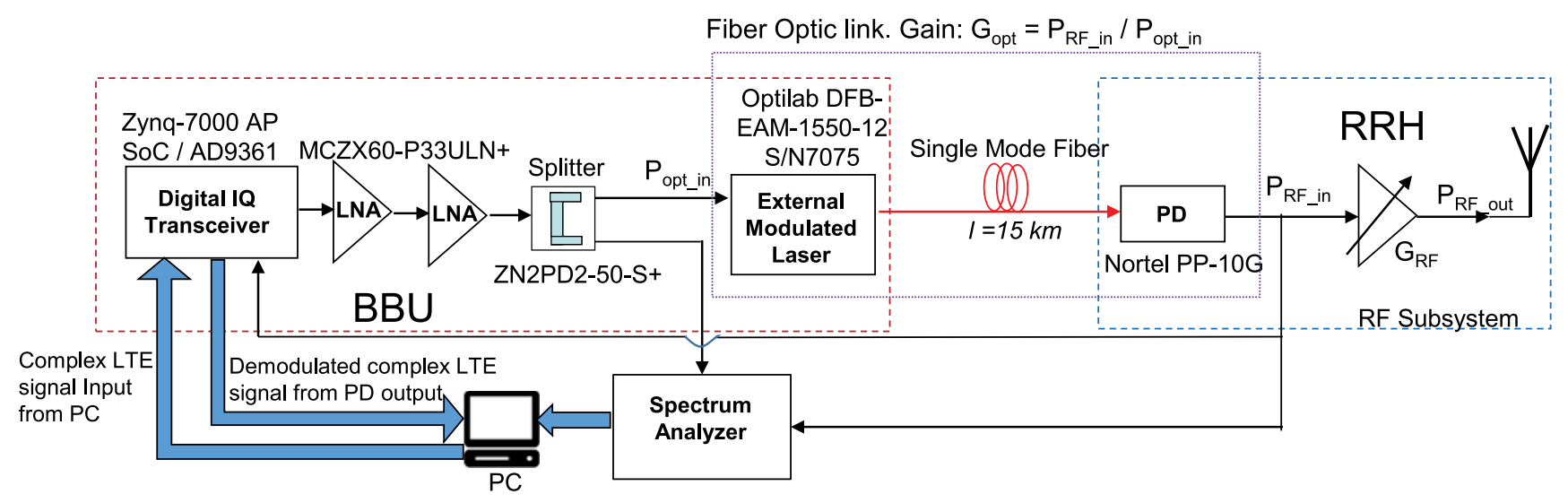

Fig. 2. Experiment set up for LTE signal transmission using RoF link.

feeding RF waveform (Direct Modulated Laser) $x_{\mathrm{DML}}(t)$ is

$$
x_{\mathrm{DML}}(t)=\operatorname{Re}\left\{e^{j \Omega_{R F} t} \sum_{\substack{k=-N / 2 \\ k \neq 0}}^{N / 2} X_{k} e^{j \Omega_{k} t}\right\}
$$

where $\Omega_{\mathrm{RF}}=2 \pi f_{\mathrm{RF}}$, and $f_{\mathrm{RF}}$ is the RF carrier frequency. Previous studies have assumed that the OFDM signal is a stationary stochastic process with a constant spectral density and averaged power. According to the previous section, the actual situation is far from a fully occupied OFDMA signal. In order to describe a more realistic scenario, the time structure and the transmitted symbols should be carefully taken into account. The transmitted signal in each LTE subframe is described by a resource grid of $N=N_{\mathrm{RB}}^{\mathrm{DL}} N_{\mathrm{sc}}^{\mathrm{RB}}$ used subcarriers $\left(N_{\mathrm{RB}}^{\mathrm{DL}}\right.$ is the number of PRBs, which depends on the downlink configured transmission bandwidth, and $N_{\mathrm{sc}}^{\mathrm{RB}}=12$, the number of subcarriers per PRB) and $N_{\text {symb }}^{\mathrm{DL}}$ the OFDM symbols in the time domain [12]. Each element in the resource grid can be uniquely identified by the index pair $(k, l)$ in a subframe where $k=0, \ldots, N_{\mathrm{RB}}^{\mathrm{DL}} N_{\mathrm{sc}}^{\mathrm{RB}}-1$ and $l=0, \ldots, N_{\mathrm{symb}}^{\mathrm{DL}}-1$, being $X_{k, l}$ the modulated symbol which corresponds with the $(\mathrm{k}, \mathrm{l})$ resource element $(\mathrm{RE})$. Thus, the LTE baseband signal needs to be more precisely described according to [12]. The time-continuous signal $x_{l}^{\mathrm{LTE}}(t)$ in OFDMA symbol $l$ in a downlink subframe is defined by

$$
x_{l}^{\mathrm{LTE}}(t)=\sum_{\substack{k=-N / 2 \\ k \neq 0}}^{N / 2} X_{k, l} \cdot e^{j 2 \pi k \Delta f\left(t-N_{\mathrm{CP}, l} \cdot T_{s}\right)}
$$

with $0 \leq t<\left(N_{\mathrm{CP}, l}+N\right) \times T_{\mathrm{S}}$ being $N_{\mathrm{CP}, 1}$ the length of the cyclic prefix in the symbol $l, N=2048$ the number of samples of useful symbols and $T_{\mathrm{S}}=1 /(15000 \cdot 2048)$ the basic time unit, for a $\Delta f=15 \mathrm{kHz}$ subcarrier spacing. Note that different OFDM symbols within a subframe have different cyclic prefix lengths. The OFDMA symbols in a subframe shall be transmitted in increased order of $l$, starting with $l=0$, where OFDM symbol $l>0$ starts at time $\sum_{l^{\prime}=0}^{l-1}\left(N_{\mathrm{CP}, l^{\prime}}+N\right) T_{\mathrm{s}}$. Finally, the feeding RF signal is

$$
\begin{aligned}
x_{\mathrm{DML}, 1}^{\mathrm{LTE}}(t)= & \sum_{\substack{k=-N / 2 \\
k \neq 0}}^{N / 2}\left|X_{k, l}\right| \cos \left[\left(\Omega_{R F}+\Omega_{k}\right)\right. \\
& \left.\times\left(t-N_{\mathrm{CP}, l} T_{\mathrm{S}}\right)+\varphi_{x k, l}\right]
\end{aligned}
$$

There are certain resource elements (corresponding with reference signals) which are always transmitted in the system whereas the remaining components (control and data) may be occupied or not, and thus, are the source which contribute to the largest variation on laser modulation. Let us define the set of RE index corresponding to the reference signals (CRS) as

$$
\mathbf{R}=\left\{(k, l), \text { s.t. } X_{k, l} \text { corresponds to ref. symbol }\right\}
$$

which is a subset of the complete resource grid symbol index set, denoted as $\mathbf{G}$. Thus, the set $\mathbf{G}-\mathbf{R}$ denote the control, user and remaining data. Therefore, a more realistic expression of the transmitted signal is

$$
\begin{aligned}
x_{\mathrm{DML}, l}^{\mathrm{LTE}}(t)= & \sum_{(k, l) \in \mathbf{R}}\left|X_{k, l}\right| \cos \left[\left(\Omega_{R F}+\Omega_{k}\right)\left(t-N_{\mathrm{CP}, l} T_{\mathrm{S}}\right)\right. \\
& \left.+\varphi_{x k, l}\right]+\sum_{(k, l) \in \mathbf{G}-\mathbf{R}}\left|X_{k, l}\right| \cos \left[\left(\Omega_{R F}+\Omega_{k}\right)\right. \\
& \left.\times\left(t-N_{\mathrm{CP}, l} T_{\mathrm{s}}\right)+\varphi_{x k, l}\right]
\end{aligned}
$$

The first sum takes reference symbols (CRS) into account. The average power of the symbols of each subframe, corresponding to this signal, will be never zero, while the second sum is the contribution of the remaining data, which ranges from almost zero if no data traffic is transmitted up to the full load conditions. Consequently, from a stochastic perspective the signal is no longer a stationary process, but rather a time-variant process whose power changes depending on the allocated resources. Thus, the transmitted signal comprises two factors, the CRS signal $x_{\mathrm{DML}, 1}^{\mathrm{LTE}, \mathrm{CRS}}(t)$ and the data $x_{\mathrm{DML}, 1}^{\mathrm{LTE}, \text { data }}(t)$

$$
x_{\mathrm{DML}, 1}^{\mathrm{LTE}}(t)=x_{\mathrm{DML}, 1}^{\mathrm{LTE}, \mathrm{CRS}}(t)+x_{\mathrm{DML}, 1}^{\mathrm{LTE}, \mathrm{data}}(t)
$$


From the laser modulation point of view, the waveform is scaled and a DC offset is added to operate the optical source. Neglecting the transmitter noise and linear distortion, the input current to the laser is given by

$$
i_{\mathrm{DL}, 1}(t)=i_{\mathrm{bias}}\left(1+\mu x_{\mathrm{DML}, 1}^{\mathrm{LTE}}(t)\right)
$$

where $\mu$ is the modulation index and $i_{\text {bias }}$ is the bias current. Using (7), the actual input current is

$$
\begin{aligned}
i_{\mathrm{DL}, \mathrm{l}}(t) & =i_{\mathrm{bias}}\left\{1+\mu\left(x_{\mathrm{DML}, \mathrm{l}}^{\mathrm{LTE}, \mathrm{CRS}}(t)+x_{\mathrm{DML}, \mathrm{l}}^{\mathrm{LTE}, \mathrm{data}}(t)\right)\right\} \\
& =i_{\mathrm{bias}}+i_{l}^{\mathrm{LTE}, \mathrm{CRS}}(t)+i_{l}^{\mathrm{LTE}, \text { data }}(t)
\end{aligned}
$$

The output optical field which feeds the fiber at a $\lambda_{0}$ wavelength is

$$
E(t, z=0)=\sqrt{P_{\mathrm{LTE}}(t)} e^{j \phi_{l d}(t)} e^{j \frac{2 \pi c}{\lambda_{0}} t}
$$

where $P_{\mathrm{LTE}}(t)$ and $\phi_{l d}$ depend on the input laser current and can be estimated by solving the laser rate equations [13]. In this work, the evaluation of the effects will be experimentally carried out. The received intensity at the photodiode output $I_{p h}(t)$ is computed from the optical field $E(t, z=L)$ at the output of the fiber using the responsivity $\Re$ and the Volterra Kernels $H_{q}(\cdot)$, [14]

$$
\begin{aligned}
I_{p h}(t) & =\Re|E(t, z=L)|^{2} \\
& =\Re\left|\sum_{q=0}^{\infty} H_{q}(E(t, z=0))\right|^{2}+n_{\mathrm{opt}}(t)
\end{aligned}
$$

The variance of receiver noise current $n_{\mathrm{opt}}(t)$ can be modelled as an AWGN model. It includes mainly thermal noise and shot noise. Alternatively, the output optical field can be calculated with the aid of numerical methods applied to the nonlinear Schrödinger equation [15]. The key point is that Radio Resource Management bears on the input laser current and the optical power. Therefore, dispersion, noise and nonlinear behavior should be different depending on the traffic load, whose impact on performance will be addressed by experimental measurements.

\section{EXPERIMENTAL RESULTS}

In this work, the variation of the distortion has been experimentally evaluated as a function of the traffic load of the cell corresponding to different maximum input power to the laser and its bias current values. The root-mean-square error vector magnitude $(E V M)$ parameter measures the in-band distortion in this paper. Performance requirements are defined in the standard [7], where it is established that the EVM of each E-UTRA carrier for different modulation schemes on Physical Downlink Shared Channel (PDSCH) should be better than $17.5 \%$ for QPSK, $12.5 \%$ for 16QAM, 8\% for 64QAM and 3.5\% for 256QAM. The optical link operation parameters (mainly the bias current and the maximum input power to the laser) must be carefully selected so that its contribution to the global distortion is the least possible. This choice stems from the fact that these EVM bounds should not be exceeded when all sources of distortion (as a result of the optical link and the RF subsystem) are included and that the main cause of non-linear distortion is the RF power amplifier (HPA). However, very conservative values of the maximum input power to the laser decrease the delivered power to the RF subsystem in the RRH more than necessary, requiring a higher HPA gain. Therefore, it is required to find a tradeoff between the distortion which introduces the optical link and the requested demands to the HPA according to power variations due to the decisions of radio resource allocations in every $1 \mathrm{~ms}$. LTE subframe.

\section{A. Experimental Setup}

The complete experimental test bench is shown in Fig. 2 through an equivalent block diagram including the real RF and optical elements. As we focus on evaluating the effects of the fronthaul FO link, the RF subsystem in the RRH is not included in the distortion analysis, but is shown in the figure to represent overall transmission system. The digital development platform used for the implementation of digital signal processes and the digital I/Q modulator and demodulator consists of a main board (ZedBoard featuring Zynq 7020 All-Programmable SoC) connected to a PC, which controls a high speed analog module with an integrated RF agile transceiver, the Analog Devices ADFMCOMMS2-EBZ. It comprises a RF $2 \times 2$ transceiver with integrated 12-bit DACs and ADCs and has a tunable channel bandwidth (from $200 \mathrm{kHz}$ to $56 \mathrm{MHz}$ ) and RX gain control. The output signal of the DAC is preamplified and upconverted to a RF frequency of $1.8 \mathrm{GHz}$, within the Band 9 of the LTE standard for FDD duplex mode. The generated signal corresponds to a LTE downlink signal (OFDM modulation) with M-QAM modulated subcarriers and $20 \mathrm{MHz}$ bandwidth. The waveform, amplified by two low noise amplifiers (Mini Circuits ZX60P33ULN+), feeds a laser diode module. An electro-absorption modulator (EAM) Distributed Feed-Back (DFB) (Optilab DFBEAM-1550-12 S/N7075), whose wavelength is $1550 \mathrm{~nm}$ is used in the experiments. The link between BBU and RRH is a singlemode ber $(\mathrm{SMF})$ with an attenuation of $0.25 \mathrm{~dB} / \mathrm{Km}$, a dispersion of $18 \mathrm{ps} /(\mathrm{nm} \cdot \mathrm{Km})$ and is $15 \mathrm{~km}$ length.

The RRH side consists of a photodetector (PD) with a responsivity of 0.9 A/W (Nortel Networks PP-10G). The PD comprises a wideband InGaAs PIN detector and a GaAS HBT preamplifier so that a great bandwidth is achieved $(11 \mathrm{GHz})$ which makes it suitable for receiving LTE signals at the appropriate frequency bands. The transimpedance gain is about 500 Ohms and the dark current takes a value of $10 \mathrm{nA}$.

In order to evaluate only the the optical link distortion, i.e. without influence of that due to the RF power amplifier, the signal is demodulated in the ADFMCOMMS2-EBZ after the optic-electric conversion and analyzed in the computer. A Spectrum Analyzer is used to visualize the generated signal spectrum in real time and to measure the signal power at the laser input and at the photodetector output.

\section{B. Results}

A set of experiments are accomplished in order to evaluate the effects of the power dynamics coming out from practical 
radio resource allocation implementations and requirements at the wireless interface of LTE-A.

Fig. 3 shows the EVM (\%) and the gain of the optical link $\left(G_{\mathrm{opt}}=P_{\mathrm{RF} \_ \text {in }} / P_{\mathrm{opt} \_ \text {in }}\right.$ in Fig. 2) in the case of several bias and maximum power configurations at the input of the RoF subsystem $\left(P_{\text {opt_in_max }}\right.$ values from $-14.5 \mathrm{dBm}$ to $5.5 \mathrm{dBm}$ modified in steps of $1 \mathrm{~dB}$ (named FO maximum input power in the figures) together with the maximum output power at the RF antenna connector when all REs are occupied. The maximum available output power at the antenna port is constrained by the specification corresponding to different coverage configurations [7]. Usual suggested values are $P_{\mathrm{RF} \text { _out }}=30 \mathrm{dBm}$ (optionally $24 \mathrm{dBm}, 37 \mathrm{dBm}$ ) for small cells and around $43 \mathrm{dBm}$ to $46 \mathrm{dBm}$ for macrocells. Once the level of $P_{\mathrm{RF} \text { _out }}$ is set according to coverage specifications (for example, $43 \mathrm{dBm}$ for $20 \mathrm{MHz}$ bandwidth is a reasonable scenario), the study shows that the smaller the RoF subsystem input power, the lower the received power at the input of the RF subsystem in the RRH and, thus, the greater the design challenges and requirements for that RF subsystem, particularly for the HPA implementation. As a result of the variation of the number of occupied PRBs (NPRBs) from $10(10 \%)$ to $100(100 \%)$ in every maximum $P_{\text {opt_in_max }}$ configuration, the input power at the RoF subsystem varies dinamically every $1 \mathrm{~ms}$, remaining the power spectral density constant according to the 3GPP specification.

The test-signal which is used in the experiments follows the LTE frame structure, consisting of different physical signal and channels including PDSCH, PDCCH, RS and synchronization data. Fig. 3 shows the obtained results with the allocated PRBs on the PDSCH when user information and 16QAM are employed. The cases of QPSK and 64QAM have been also evaluated with similar conclusions. The analysis of the influence of the laser bias current proofs that values around $50 \mathrm{~mA}$ offer the best results. Furthermore, a maximum $P_{\text {opt_in }}$ around $P_{\text {opt_in_max }}=-5.5 \mathrm{dBm}$ yields to the lowest $E V M$ values (below $4.5 \%$ ) for any PRB allocation configuration with almost constant gains $\left(G_{\mathrm{opt}}=-17 \mathrm{~dB}\right)$. This scenario could be a suitable value for the optical link configuration. However it is possible to obtain near-minimum distortion values with higher laser input powers when the traffic load is less than $100 \%$, which is the most probable, as shown in Fig. 4 where a detailed comparison is performed for the most attractive values ( $E V M$ rises when $P_{\text {opt_in_max }}$ is lower than $-6.5 \mathrm{dBm}$ due to noise, whereas nonlinear distortions are very significant above $-1.5 \mathrm{dBm}$ ). For example, according to Fig. 4(a), if we set the maximum distortion due to the optical link in $E V M=5 \%$, for occupations of PRBs up to $55 \%$ the input power to the laser up to $-1.5 \mathrm{dBm}$ can be increased; for occupations between $56 \%$ and $67 \%$, the system could be configured to use a laser input power of $-2.5 \mathrm{dBm}$; for occupations between $68 \%$ and $82 \%$ that might be $-3.5 \mathrm{dBm}$, etc. Each increase of $1 \mathrm{~dB}$ in the RF input power results in $1 \mathrm{~dB}$ less in the HPA gain, which is clearly an advantage. In addition we evidence that similar input power values corresponding to different power spectral densities (for example, $P_{\text {opt_in }}=-6.26 \mathrm{dBm}$ associated to $P_{\text {opt_in_max }}=-1.5 \mathrm{dBm}$ and $N P R B s=30$ vs. $P_{\text {opt_in }}=P_{\text {opt_in_max }}=-6.5 \mathrm{dBm}$ when
$N P R B s=100$ are occupied) lead to very different $E V M$ values. Fig. 4(b) shows the variations of the input power at the RF subsystem as a result of the variations of occupied PRBs (NPRBs) in cases with the same range of values of $P_{\text {opt_in_max }}$. The power amplifier must work in the linear operation region in the whole range of variation of the RF input power. The required gain in the HPA thus remains constant for any load condition, once $P_{\text {opt_in_max }}$ is fixed. Nevertheless, if we focus on the RF input power when $N P R B=100$, the required gain for $P_{\text {opt_in_max }}=-1.5 \mathrm{dBm}$ in order to reach $P_{\mathrm{RF} \text { _out_max }}(30 \mathrm{dBm}$ in small cells or $46 \mathrm{dBm}$ in macrocells for example) is about $4 \mathrm{~dB}$ lower than that required for $P_{\text {opt_in_max }}=-5.5 \mathrm{dBm}$.

The results of this work do not only determine the proper values of several parameters from the optical link and the radiofrequency subsystem gain, but also can be taken into account to achieve a more optimal design of both physical and link layers mechanisms in C-RAN architectures. Evaluation studies of both optical and RF subsystems in analog radio over fiber systems usually do not take into account the operation of higher levels of the wireless system. Results suggest improvements on the scheduler strategy design process (which must decide how many PRBs are assigned in the transmission to the UEs) to minimize the distortion introduced by the optical link. Being the normal operation of the BS far from full load conditions, the scheduling strategies can be developed in order to balance the number of allocated PRBs through the time (by scheduling similar number of PRBs every TTI), instead of gathering these on few subframes within the frame. If the service delay requirements allow it (delays of few milliseconds $(2,3,4)$ are tolerable in all the cases) it may be more beneficial for the scheduler to make PRB allocation decisions looking for a similar percentage of occupancy in all subframes instead of minimizing to the extreme (reduce by 1,2 o $3 \mathrm{~ms}$ ) the waiting time in the transmission buffer if this supposes a high variability of the PRB occupation in subsequent subframes. Figs. 5 and 6 show experimental EVM performance, constellations diagrams and power spectral density functions for an example that illustrates the potential benefits. Setting an input power of $-5.5 \mathrm{dBm}$ for a bias of $50 \mathrm{~mA}$, with $100 \mathrm{PRBs}$ occupied in a subframe we have an $E V M$ of $4,36 \%$, and for 20 occupied PRBs in the next subframe we have an $E V M$ of $2,01 \%$, whereas if we occupy 60 PRBs in the two subframes, we have in both an EVM of 2,91\%. Moreover, if all the time we have about $50 \%-60 \%$ of occupied PRBs, we can raise the input power to $-2.5 \mathrm{dBm}$ keeping the $E V M$ below $4.58 \%$. That is, higher maximum input power values (for example, $-2.5 \mathrm{dBm}$ ) can be chosen, being the link budget improvement about $3 \mathrm{~dB}$, from the HPA gain requirement point of view.

Moreover, in LTE network deployments, it is very convenient that the radio resource allocation patterns are as stable as possible in order to reduce the interference power variation. Thus, in fact, many of the implementations currently seek uniform distributions of the PRB allocations instead of high variable allocations. Although fast adaptation (every $1 \mathrm{~ms}$ ) of the operating point of the RF amplifier can be complicated, it is not necessary to implement it, since the variations in the traffic load are much 


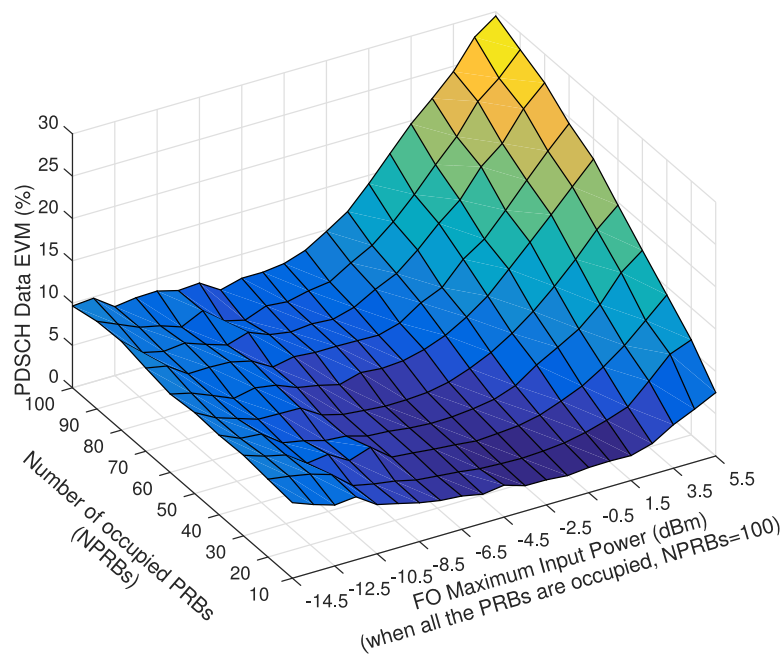

(a)

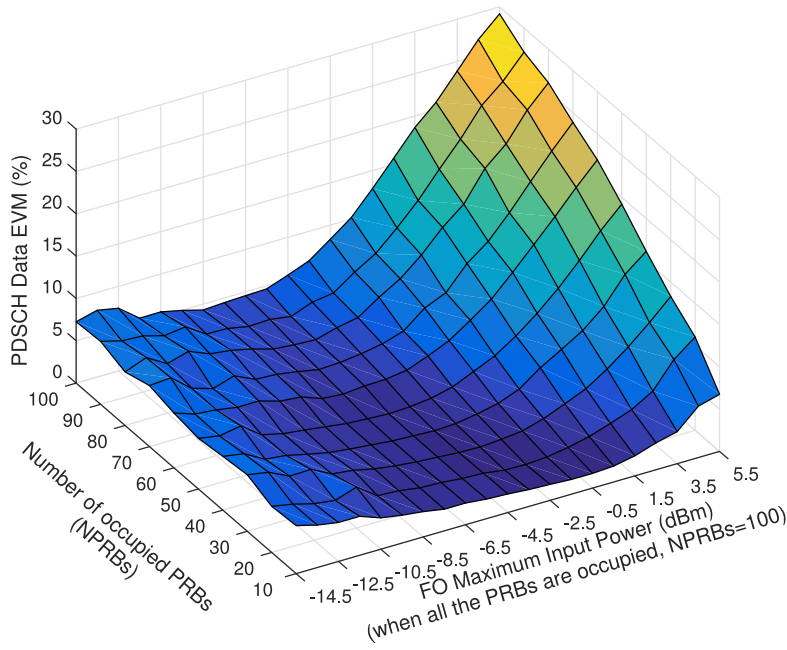

(c)

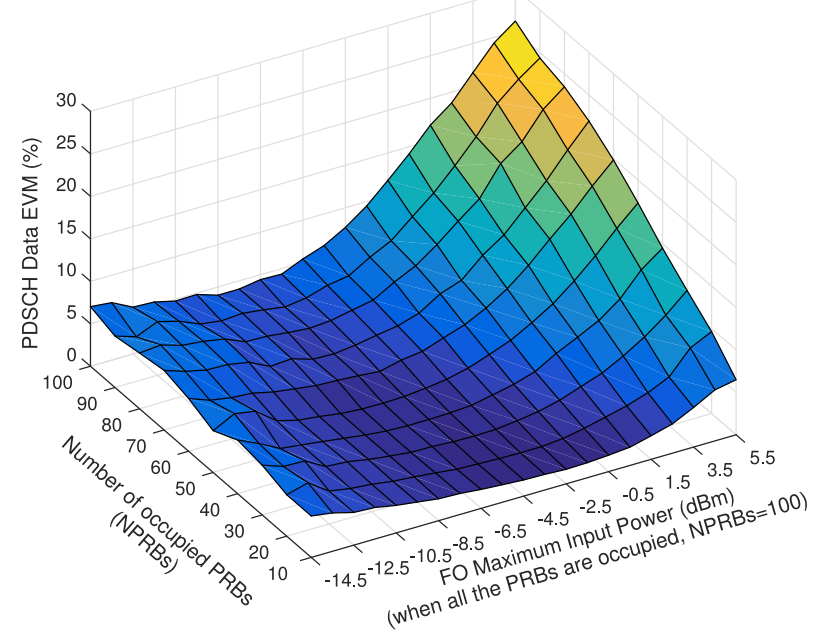

(e)

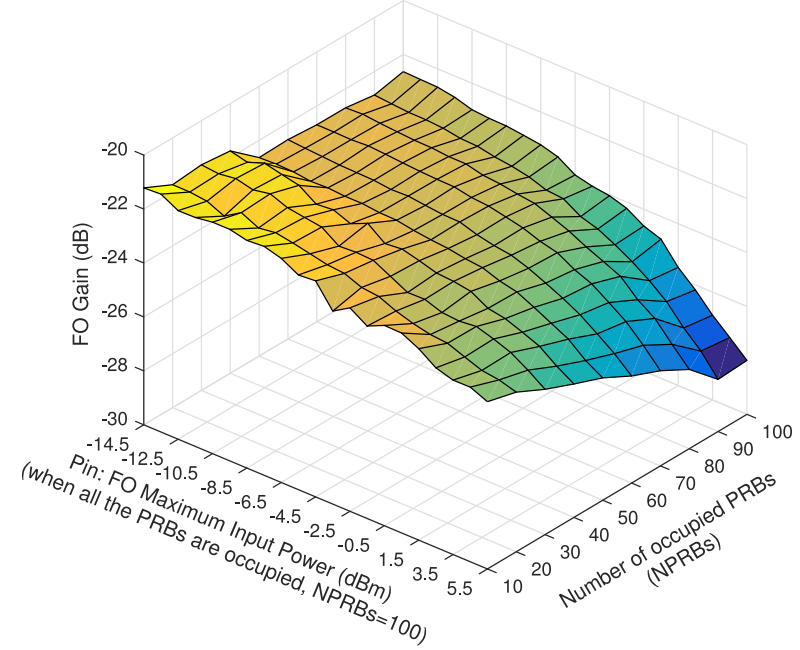

(b)

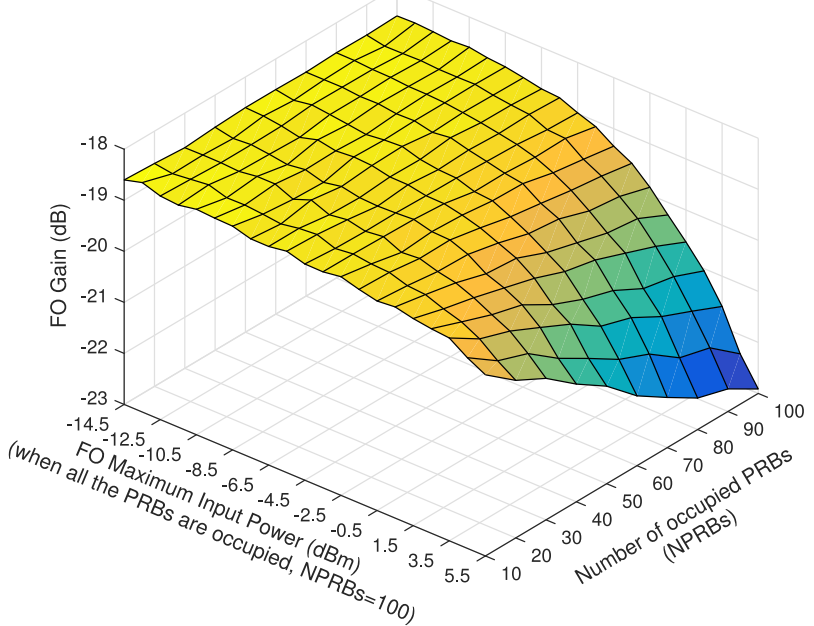

(d)

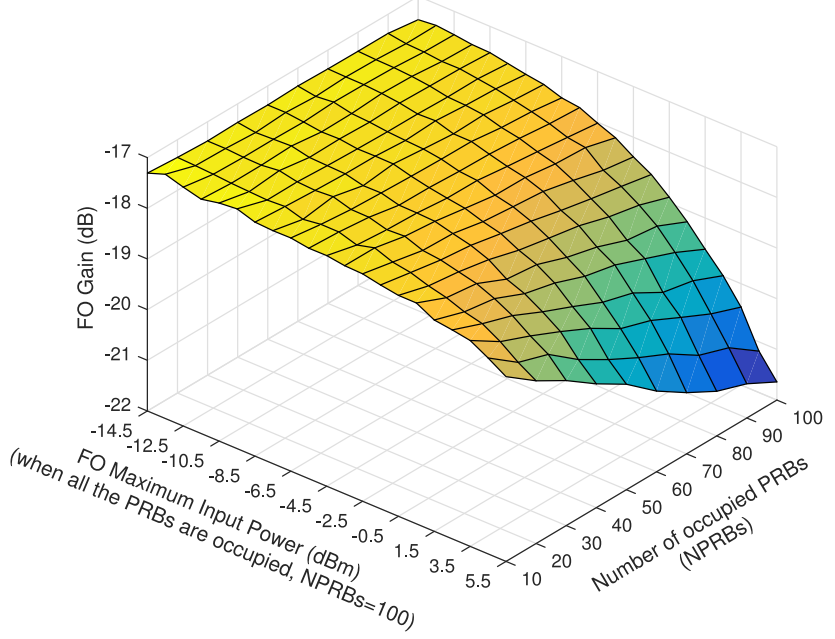

(f)

Fig. 3. PDSCH Data $E V M(\%)$ and FO Gain for several maximum power configurations at the input of the RoF subsystem $\left(P_{\mathrm{opt} \text { _in_max }}\right)$ and a variable number of allocated PRBs (corresponding with variable $P_{\mathrm{opt} \text { in }}$ ), considering several bias values. (a) Case a1: $E V M$ bias $30 \mathrm{~mA}$. (b) Case b1: Gain bias $30 \mathrm{~mA}$. (c) Case a2: $E V M$ bias $40 \mathrm{~mA}$. (d) Case b2: Gain bias $40 \mathrm{~mA}$. (e) Case a3: $E V M$ bias $50 \mathrm{~mA}$. (f) Case b3: Gain bias $50 \mathrm{~mA}$. 


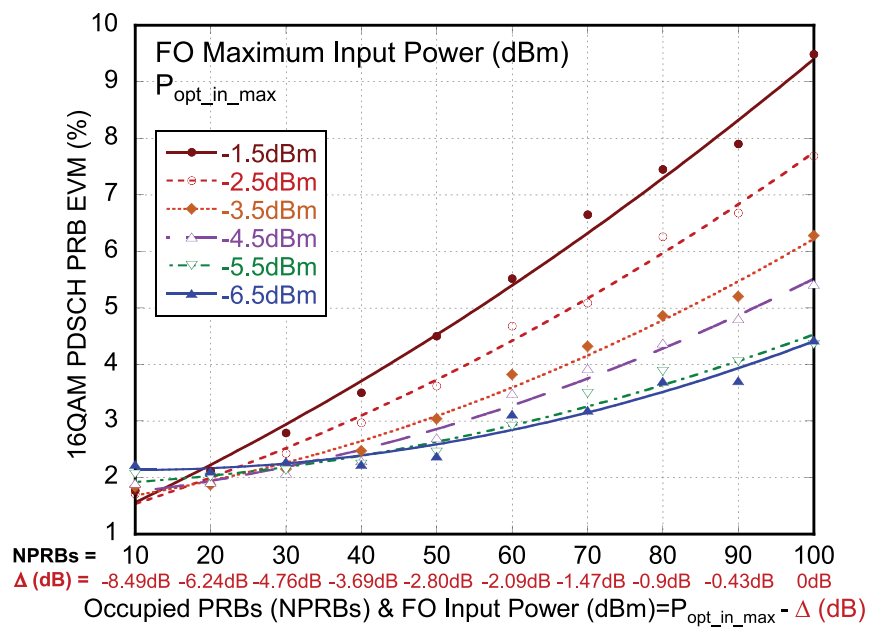

(a)

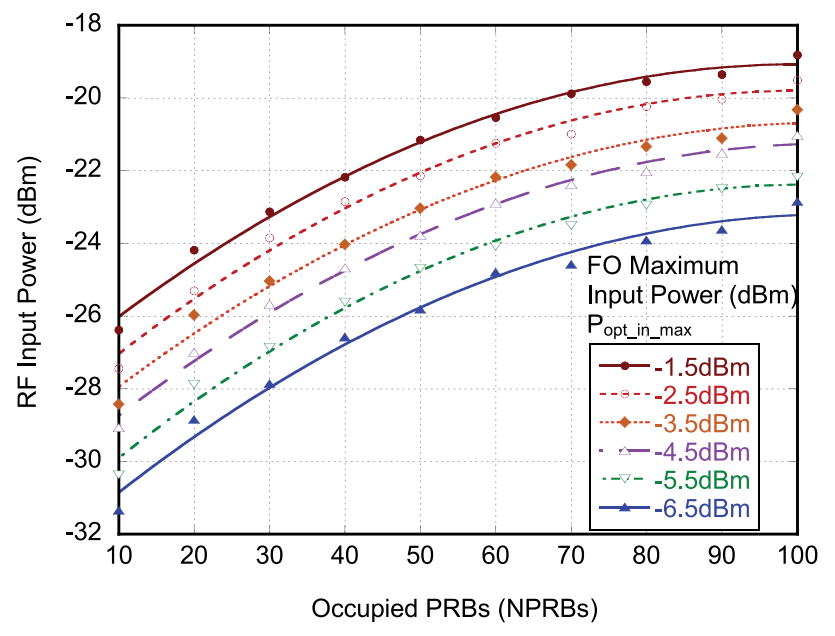

(b)

Fig. 4. Detailed comparison of $E V M$ and achieved RF Input power for several FO input power and number of allocated PRBs. (a) $E V M$ (\%) for bias $50 \mathrm{~mA}$. (b) Achieved RF Input Power (dBm).

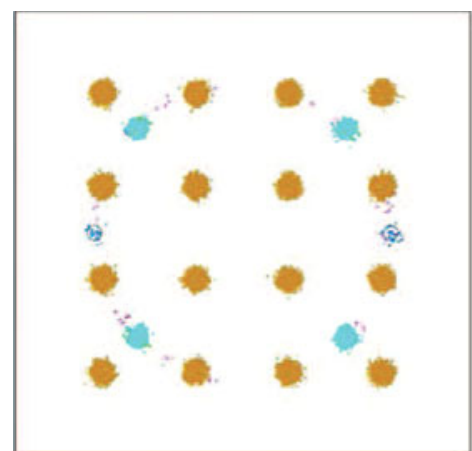

(a1) $\mathrm{NPRBs}=100, \mathrm{EVM}=4.35 \%$

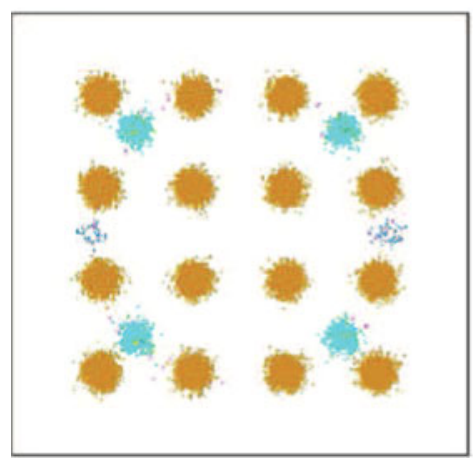

(b1) $\mathrm{NPRBs}=100, \mathrm{EVM}=7.69 \%$

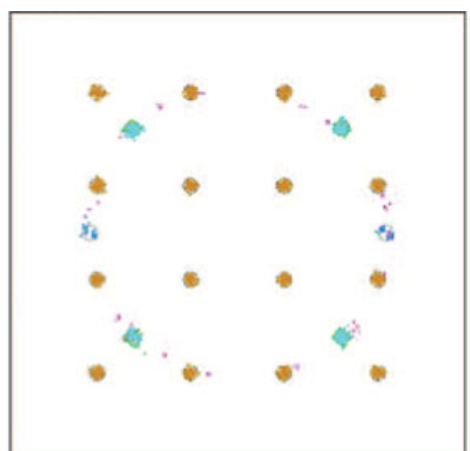

(a2) $\mathrm{NPRBs}=20, \mathrm{EVM}=2.01 \%$

(a)

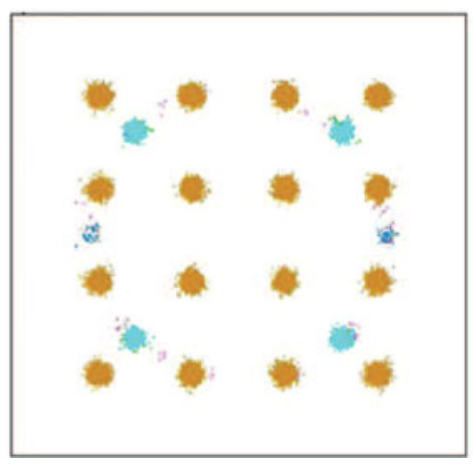

(b2) $\mathrm{NPRBs}=60, \mathrm{EVM}=4.58 \%$

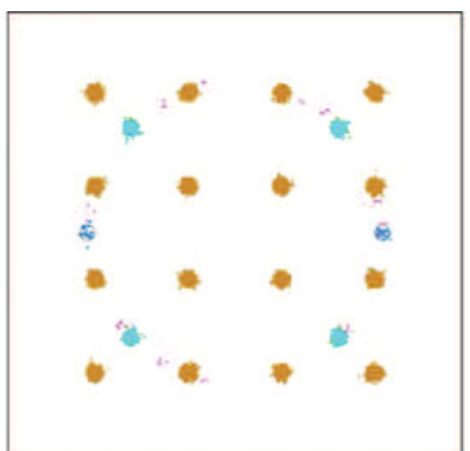

(a3) $\mathrm{NPRBs}=60, \mathrm{EVM}=2.91 \%$

(b)

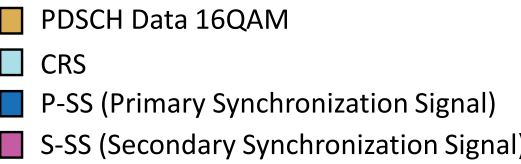

Fig. 5. Experimental received constellations in particular cases with different number of allocated PRBs. (a) EVM for FO Maximum Input Power configuration (Popt_in_max) of $-5.5 \mathrm{dBm}$, BIAS $=50 \mathrm{~mA}$. (b) EVM for FO Maximum Input Power configuration (Popt_in_max) of $-2.5 \mathrm{dBm}$, BIAS = 50 mA.

slower. In a cell, the average load keeps practically constant for minutes and even hours and very rarely the cell will be fully loaded. Besides, in a shorter scale, an on-time concurrence of several services results on PRB demand requirements that remains constant for at least tens-hundreds of seconds. Thus, the adaptation is feasible. Besides, if most of the time the cell has a traffic load of, for example, 50\%, an optical input power of $-2.5 \mathrm{dBm}$ can be set or even $-1.5 \mathrm{dBm}$, relaxing the RF amplifier gain requirements in $3 \mathrm{~dB}-4 \mathrm{~dB}$. If a bias current of $40 \mathrm{~mA}$ is considered, the RF amplifier gain can be relaxed in $4 \mathrm{~dB}$. When the traffic load increases, we should simply reduce the optical input power and increase the gain of the RF amplifier. Finally, 


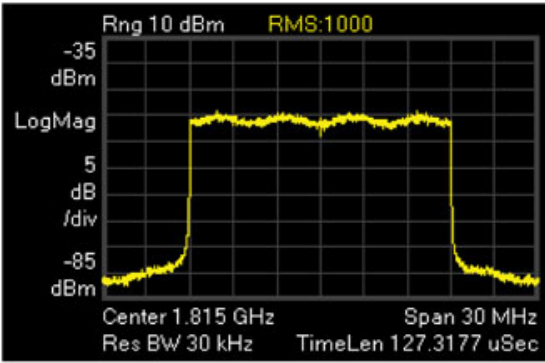

(a1) NPRBs $=100$

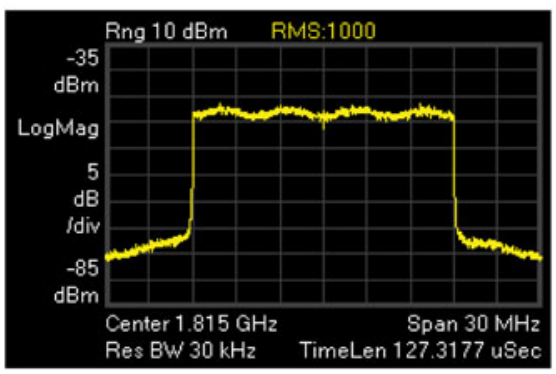

(b1) NPRBs $=100$

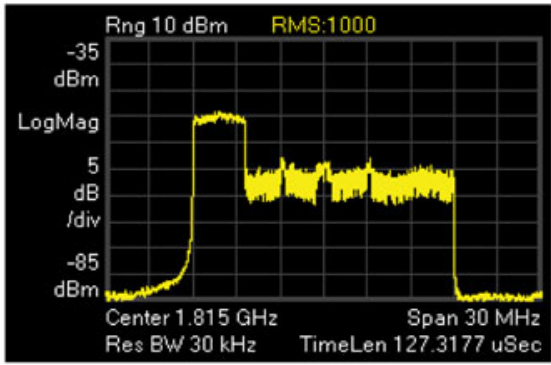

(a1) $\mathrm{NPRBs}=20$

(a)

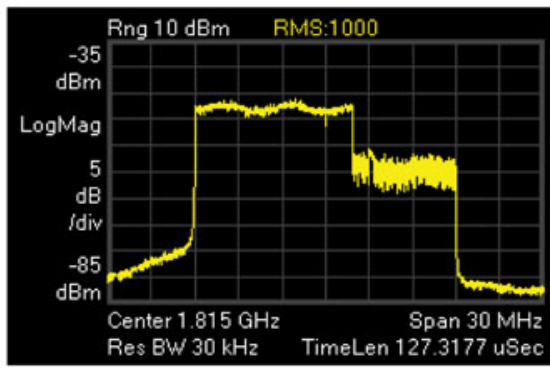

(b1) NPRBs $=60$

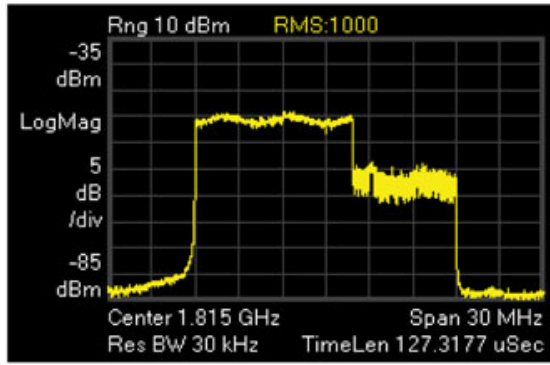

(a1) $\mathrm{NPRBs}=60$

(b)

Fig. 6. Experimental Power Spectral Density in particular cases with different number of allocated PRBs. (a) Power Spectral Density for FO Maximum Input Power configuration (Popt_in_max)) of $-5.5 \mathrm{dBm}$, BIAS $=50 \mathrm{~mA}$. (b) Power Spectral Density for FO Maximum Input Power configuration (Popt_in_max) of $-2.5 \mathrm{dBm}, \mathrm{BIAS}=50 \mathrm{~mA}$.

we note that if the system is practically always running with a stable load of $20 \%-50 \%$, an optical setting based on measurements made at full load results is a suboptimal configuration, clearly improvable.

\section{CONCLUSION}

In this work, we have experimentally evaluated the distortion introduced by the optical link as a result of the variations in the RF input power due to fluctuations in the traffic load. We have verified that it is possible to set the bias current to a value which leads to low distortion in the entire range of number of occupied radio resources. Despite, results show that the laser input power choice only considering the distortion below a certain threshold for $100 \%$ radio resource occupation is highly inefficient under usual system operation conditions. Adaptively varying the RF input power depending on the number of occupied radio resources may reduce the requested RF high power amplifier gain up to $4 \mathrm{~dB}$, thereby increasing its efficiency or reducing the nonlinear distortion it causes in the transmitted signal.

\section{REFERENCES}

[1] T. Pfeiffer, "Next generation mobile fronthaul and midhaul architectures," J. Opt. Commun. Netw., vol. 7, no. 11, pp. B38-B45, 2015.

[2] N. Alic, "Cancellation of nonlinear impairments in ber optic transmission systems," Opt. Fiber Commun. Conf., 2016, Paper Tu2E.1.

[3] P. T. Dat, A. Kanno, N. Yamamoto, and T. Kawanishi, "Full-duplex transmission of LTE-A carrier aggregation signal over a bidirectional seamless fiber-millimeter-wave system," J. Lightw. Technol., vol. 34, no. 2, pp. $691-$ 700, Jan. 2016.

[4] Y. Fan, "Full-duplex transmission of IEEE 802.11ac-compliant MIMO WLAN signals over a 2-km 7-core fiber," Chin. Opt. Lett., vol. 15, no. 1, pp. 010011-10015, Jan. 2017.
[5] S.-H. Cho, C. Han, H. S. Chung, and J. H. Lee, "Demonstration of mobile fronthaul test bed based on RoF technology supporting two frequency assignments and $2 \times 2$ MIMO antennas," ETRI J., vol. 37, no. 6, pp. 10551064, Dec. 2015.

[6] S. E. Alavi, M. R. K. Soltanian, I. S. Amiri, M. Khalily, A. S. M. Supaat, and H. Ahmad, "Towards 5G: A photonic based millimeter wave signal generation for applying in 5G access fronthaul," Scientific Rep., vol. 6, 2016, Art. no. 19891.

[7] 3rd Generation Partnership Project; Technical Specification Group Radio Access Network; Evolved Universal Terrestrial Radio Access (E-UTRA); Base Station (BS) Radio Transmission and Reception (Release 14), 3GPP TS 36.104 V14.2.0 (2016-12), 2016.

[8] F. Capozzi, G. Piro, L. A. Grieco, G. Boggia, and P. Camarda, "Downlink packet scheduling in LTE cellular networks: Key design issues and a survey," IEEE Commun. Surveys Tut., vol. 15, no. 2, pp. 678-700, May 2013.

[9] T. Kanesan, W. P. Ng, Z. Ghassemlooy, and C. Lu, "Investigation of optical modulators in optimized nonlinear compensated LTE RoF system," $J$. Lightw. Technol., vol. 32, no. 10, pp. 1944-1950, May 2014.

[10] W. P. Ng, T. Kanesan, Z. Ghassemlooy, and C. Lu, "Theoretical and experimental optimum system design for LTE-RoF over varying transmission span and identification of system nonlinear limit," IEEE Photon. J., vol. 4, no. 5, pp. 1560-1571, Oct. 2012.

[11] C. Sánchez, J. Wei, B. Ortega, and J. Capmany, "Comprehensive impairment and performance description of directly modulated/detected OOFDM systems," J. Lightw. Technol., vol. 31, no. 20, pp. 3277-3288. Oct. 2015.

[12] 3rd Generation Partnership Project; Technical Specification Group Radio Access Network; Evolved Universal Terrestrial Radio Access (EUTRA); Physical Channels and Modulation (Release 14), 3GPP TS 36.211 V14.3.0 (2017-06), 2017.

[13] I. Fatadin, D. Ives, and M. Wicks, "Numerical simulation of intensity and phase noise from extracted parameters for CW DFB lasers," J. Quantum Electron., vol. 42, no. 9, pp. 934-941, Sep. 2016.

[14] K. V. Peddanarappagari and M. Brandt-Pearce, "Volterra series transfer function of single-mode fibers," J. Lightw. Technol., vol. 15, no. 12, pp. 2232-2241, Dec. 1997.

[15] Q. Zhang and M. I. Hayee, "Symmetrized split-step fourier scheme to control global simulation accuracy in fiber-optic communication systems," J. Lightw. Technol., vol. 26, no. 2, pp. 302-316, Jan. 2008. 
Pedro Luis Carro was born in Zaragoza, Spain, in 1979. He received the Telecommunication Engineering (M.S.) and Ph.D. degrees from the University of Zaragoza, Zaragoza, Spain, in 2003 and 2009, respectively. In 2002, he carried out the Master thesis on antennas for mobile communications at Ericsson Microwave Systems, AB in Gothemburg, Sweden. From 2002 to 2004, he was with Rymsa S.A., where he worked in the Space and Defense Department as an Electrical Engineer. From 2004 to 2005, he was with TELNET Redes Inteligentes in the Research and Development Department as an RF Engineer, involved in radio over fiber systems. In 2005, he joined the University of Zaragoza, where he is an Associated Professor with the Electronics Engineering and Communication Department. His research interests include the area of microwave photonics, mobile antenna systems, passive microwave devices, and power amplifiers.

Ángela Hernández Solana received the Telecommunication Engineering and Ph.D. degrees from the Universitat Politcnica de Catalunya (UPC), Barcelona, Spain, in 1997 and 2005, respectively. She has been with UPC and University of Zaragoza, Zaragoza, Spain, where she is an Associate Professor. She has been co-investigator of more than 45 research projects, 22 funded by public administrations (some of them as PI, focusing in LTE/LTE-A and public safety solutions) and by major industrial and mobile companies, all of them working on wireless technologies. Her research interests include $5 \mathrm{G} / 4 \mathrm{G}$ technologies, heterogeneous communication networks, and mission-critical communication networks, with emphasis on transmission techniques, radio resource management and quality of service, mobility management and planning, and dimensioning of mobile networks.
Antonio Valdovinos Bardají received the Telecommunication Engineering and Ph.D. degrees from the Universitat Politcnica de Catalunya (UPC), Barcelona, Spain, in 1990 and 1994, respectively. He has been with UPC and at the University of Zaragoza, Zaragoza, Spain, where he is a Full Professor since 2003. He has been responsible of more than 35 research projects funded by public administrations and by major industrial and mobile companies, all of them working on wireless technologies. His research interests include $5 \mathrm{G} / 4 \mathrm{G}$ technologies, heterogeneous communication networks, and mission-critical communication networks, with emphasis on transmission techniques, radio resource management and quality of service, mobility management and planning, and dimensioning of mobile networks. 